\title{
Aprendizaje experiencial mediante estudio de casos, basados en profesionales contemporáneos, en los primeros cursos de Grado.
}

\section{Francisco de la Torre Oliver ${ }^{\text {ab }}$}

a Departamento de Pintura, Universitat Politècnica de València (fratorol@pin.upv.es).

${ }^{\mathrm{b}}$ Centro de Investigación Arte y Entorno (CIAE), Universitat Politècnica de València.

\begin{abstract}
This project aims to integrate reference professionals, in our case active contemporary artists, through the methodology of case studies from an innovative and experimental approach in order to motivate the first-year students of the Degree Fine Arts.

Through this active methodology we incorporate the experience of professionals through direct contact with students in the classrooms or in the spaces of creation or/and exhibition (studies, galleries and museums).

We present, as a sample of our research, the analysis of the Sati Tena case carried out in the course 2018-19 in the subject Fundamentals of color and painting.
\end{abstract}

Keywords: Study of illustrative cases, innovation, experimental, motivation, Fine Arts, contemporary artists, active methodology

\begin{abstract}
Resumen
Este proyecto persigue integrar a profesionales de referencia en el programa de la asignatura, en nuestro caso artistas contemporáneos en activo, mediante la metodología de estudio de casos desde un enfoque innovador y experimental con el fin de motivar a los alumnos de primer curso del Grado de Bellas Artes. A través de esta metodología activa incorporamos la experiencia de los artistas a través del el contacto directo con los alumnos en las aulas y en los espacios de creación o/y exhibición (estudios, galerías y museos).

Presentamos, como ejemplo de nuestra investigación, el análisis del caso Sati Tena realizado durante el curso 2018-19 en la asignatura Fundamentos del color y de la pintura.
\end{abstract}

Palabras clave: Estudio de casos ilustrativos, innovación, experimental, motivación, metodología activa, Bellas Artes, artistas contemporáneos.

\section{Introducción}

Podría parecer anómalo que, en un grado vocacional como es Bellas Artes, a los profesores nos preocupara la motivación de nuestros alumnos. En el caso de la asignatura objeto de nuestro estudio, de carácter obligatorio e impartida en el primer curso, existirían diferentes factores que afectarían a nuestros estudiantes en este sentido. Por un lado, los propios de cada estudiante y, por otro, los que afectarían en general al grupo. 
En el caso de los alumnos que comienzan el grado con un perfil curricular muy definido, entienderían el carácter obligatorio de una asignatura como un obstáculo para alcanzar su meta, ignorando de este modo una visión transversal necesaria desde la que comprender el valor que estos contenidos aportan a sus objetivos. Por otro lado, nos enfrentaríamos a la dificultad que supone la impaciencia propia de una generación que está habituada a la instanteneidad, incompatible con un aprendizaje donde los contenidos teóricos se interiorizan mediante la práctica para, de ese modo, pasar a conformar sus claves creativas. Tampoco debemos ignorar el tamaño de los grupos a los que nos enfrentamos en las aulas en estos cursos, ya que dificultaría el seguimiento sistemático de la evolución individual de cada alumnos en el tiempo de duración de las clases.

Son estas circunstancia, entre otras, las que propiciarían la desmotivación de parte del alumnado que, al perder el ritmo de aprendizaje del grupo, requeriría una atención individualizada en tutorías personales.

Como profesores, en tanto que profesionales de la enseñanza, debemos ser capaces de actualizarnos y desarrollarnos profesionalmente con el objetivo de conectar la teoría, la técnica y la práctica (Torre, 2009, p. 8). En este sentido, nos planteamos una actitud innovadora y creativa en el desarrollo de nuestra práctica para implicar al alumno en su formación mediante actitudes flexibles, dominio de los contenidos y competencia didáctica. Al plantearnos incluir en el curriculum de la asignatura estrategias creativas, buscamos un aprendizaje más atrayente y motivador donde el alumno sea el protagonista mediante su implicación activa y la autoevaluación. De la definición que propone el profesor De la Torre (1993), destacaríamos claves tales como la predisposición a los cambios, la capacidad para integrar y relacionar experiencias aprovechando las oportunidades que nos ofrece el propio transcurso de la actualidad y, por último, nuestro interés por promover el aprendizaje por descubrimiento en un clima de seguridad y comunicación con los alumnos (Torre, 2009, p. 9). Nuestro punto de partida sería la percepción, a través del contacto diario con los alumnos, de cierta ralentización en la realización de las prácticas. Un hecho que nos conduciría a estudiar el modo de abordar la motivación positiva, ligada a sentimientos tales como el entusiasmo, la perseverancia y la confianza, para fomentar así el rendimiento. Como señala Goleman (2007), al margen de las capacidades innatas, la recompensa añadida del éxito en la vida dependerían de la motivación. Por tanto, nuestras estrategias irían orientadas a potenciar las emociones en los estudiantes para favorecer sus capacidades y, en la medida que esten motivados por el entusiasmo en lo que hacen recuperen los estímulos necesarios para el logro (p. 145).

A pesar del estudiado ritmo con el que se planifica el desarrollo de las prácticas, los alumnos acaban percibiendo cierta rutina en el día a día de su asistencia a clase. Y sería en la ruptura de estos ciclos mediante el empleo de hitos que afecten a sus emociones como consideramos que podríamos recuperar la ilusión que el alumnado manifestaba al inicio del curso. En este caso la función motivadora del profesor sería, como afirman los movimientos de renovación pedagógica, fundamental para facilitar el aprendizaje. De este modo, perseguimos despertar de nuevo el interés, pero también estilular el deseo de aprender para alcanzar los objetivos marcado en el proyecto docente de la asignatura (Sevillano, 1999).

En nuestro caso, y teniendo en cuenta el perfil de la asignatura, pensamos que poner por primera vez en contacto a un alumno de primer curso con el mundo artístico a través de sus representantes, artistas contemporáneos de reconocido prestigio ajenos al ámbito académico, 
sería un modo de reactivar la motivación de los discentes, estímulandolos para favorecer la compresión y adquisición de ideas. De modo que, al afrontar la materia desde la experiencia de artistas plásticos en activo despertaríamos la curiosidad en los estudiantes (Zabalza, 2002). Estas reflexiones son las que nos llevarían a planificar y diseñar una serie de actividades extraordinarias asociadas a las diferentes unidades didácticas con la intención, fundamentalmente, de servirnos de sus capacidades motivadoras. A lo largo de nuestra carrera como docente, y como antiguo alumno de este grado, habríamos comprobado los efectos positivos que el contacto con profesionales habría provocado en la motivación de los alumnos (Fernández, 2006, pp. 42-43).

Es en este momento cuando nos preguntamos por cual sería la metodología más adecuada para cumplir nuestros propósitos, y cómo evaluarla. Concluimos que el estudio de las soluciones llevadas a cabo por artistas contemporáneos en su obra sobre las problemáticas que les plantemos a los alumnos en clase les ofrecerían un nuevo punto de vista sobre las prácticas desarrolladas en el aula. Al diseñar nuestra metodología, deberíamos tener en cuenta que estas prácticas están basadas en los contenidos teóricos expuestos en las clases magistrales asociadas a las unidades didácticas que componen la asignatura.

$\mathrm{Al}$ acercarnos al trabajo de artistas contemporáneos, y poder conocer sus métodos de trabajo y sus claves estéticas y conceptuales de primera mano a través del contacto directo permitiría, entre otras cosas, presentar un caso real que los alumnos pueden observar e interesarse por los detalles a través de la interactuación con los autores. Pensamos que este sería un revulsivo frente al cuestionamiento de la utilidad de sus prácticas, más alla de ser el modo de superar la asignatura a través de una valoración favorable.

El estudio de casos conforma parte de las técnicas de la enseñanza, y presentados por sus autores permitiría la interactividad entre los creadores y alumnos resultando más eficaz. Si nos remontamos a las academias instauradas en el Renacimiento, observamos que estarían conformadas por grupos de artistas de diferentes edades y formación (algunos neófitos y otros artistas consumados) reunidos con el objetivo de trabajar y contemplar las demostraciones que hacían de técnicas o principios novedosos, o bien para discutir teorías del arte y otras tendencias culturales de carácter general (Efland, 2002). Estos círculos artísticos, inspirados en ideas de Leonardo da Vinci o Miguel Ángel, estarían patrocinadas por mecenas como Cosimo o Lorenzo de Medici. Una filosofía que volvemos a encontrar en experiencias como las de la escuela de la Bauhaus en los años veinte del siglo pasado, donde artistas como Itten, Albers, Kandinsky, Klee o Moholy-Nagy se involucrarían en las escuelas de arte.

Si nos trasladamos al caso que nos ocupa, también en la facultad de Bellas Artes de Valencia encontraríamos artistas de reconocido prestigio impartiendo docencia en sus aulas. Pero creemos que el efecto que obtendremos al trabajar con artistas no vinculados a la docencia reglada sería de mayor calado al encontrar los alumnos una mayor complicidad con profesionales que no perteneciesen a un sistema educativo, en el que se sentirían atrapados.

Si bien la metodología del estudio de casos ilustrativos estaría definida como una situación que va más allá de la toma de decisiones, donde se analiza un problema real y la solución que se adoptó atendiendo al contexto, permitiendo al grupo "aprender sobre la forma que una determinada organización o profesional ha tomado una decisión y el éxito de la misma" (Labrador, 2008, p. 30). Nuestro enfoque se situaría en el campo de la educación artística del arte contemporáneo, entre la historia del arte y la práctica artística actual. Podríamos

(cc) EY-NC-ND 2019, Universitat Politècnica de València 
Aprendizaje experiencial mediante estudio de casos, basados en profesionales contemporáneos, en los primeros cursos de Grado.

presentar como uno de nuestros referentes la iniciativa impulsada por el profesor José Miguel Cortes desde la dirección del Instituto Valenciano del Arte Moderno (IVAM). Desde que en el año 2015 realizara Caso de Estudio. Cuerpo, Espacio y Tiempo en Bruce Nauman con el objetivo de ir más allá de la exposición y profundizar en el estudio de la obra de un artista, planteó, los "Casos de Estudio" como proyectos que vinculan el marco expositivo, con la investigación y el análisis de la obra de artistas determinantes en el último siglo son una línea se trabajo del museo (IVAM, 2015).

Por tanto, la apuesta pedagógica por el método del caso ilustrativo incidiría como método activo en una participación constante del estudiante, tanto individualmente como en grupo, donde asumiría mayor responsabilidad en el desarrollo de la actividad y lo acercaría a la realidad de su futuro profesional (Labrador, p. 27). Y, por nuestra parte, debemos actuar como observadores neutrales registrando las actuaciones, participación, dificultades, dudas, comentarios, etc.

\section{Objetivos}

Después de plantear el contexto, podemos señalar que el objetivo general de nuestro proyecto sería integrar en el programa de la asignatura, mediante el estudio de casos, a profesionales de referencia en el campo de la materia a impartir -en nuestro caso, artistas contemporáneos en activo- con el fin de motivar a los alumnos a través de metodologías activas.

Entenderemos el estudio de casos ilustrativos desde un enfoque innovador y experimental, incorporando la experiencia de los profesionales a través del contacto directo con los alumnos en las aulas o en los espacios de creación o/y exhibición (estudios, galerías y museos).

En cuanto a los objetivos específicos necesarios para llevar a cabo nuestros propósitos, señalaremos:

Diseñar una metodología innovadora basada en el caso ilustrativo desde planteamientos experimentales.

Introducir en la academia/universidad el discurso de los artistas, a través de la colaboración de profesionales referentes en cada una de las materias.

Obtener evidencias del grado de motivación y aprendizaje del estudio de casos en los alumnos.

Ofrecer los resultados de nuestra experiencia a la comunidad universitaria a través de diferentes medios.

Confeccionar material didáctico digital a partir de la documentación del estudio de casos ilustrativos con el fin de cumplir contenidos curriculares de carácter motivador en el programa docente de la asignatura.

\section{Desarrollo de la innovación}

Como hemos expuesto anteriormente, trabajaríamos con casos ilustrativos asociados a unidades didácticas de la asignatura. El primer paso que realizamos fue analizar el contenido de la asignatura e identificar cuales serían las fases que precisaban un refuerzo motivacional y comenzar a implementar la metodología en estas unidades didácticas.

Fundamentos del color y de la pintura es una asignatura de carácter eminentemente práctico que se imparte en el módulo 1 del grado de Bellas Artes, Principios básicos para la formación 
artística, y su principal objetivo es la adquisición de conocimientos fundamentales acerca del color en sus aspectos físico-perceptivo, cultural y artístico. Al tratarse de una asignatura de iniciación, donde no se requieren conocimientos previos, nos encontramos ante un alumnado con importantes diferencias respecto a sus conocimientos y habilidades, un factor que resulta determinante a la hora de elaborar el proyecto docente de la asignatura.

La heterogeneidad del medio centenar de alumnos que componen los grupos aporta una innegable riqueza al conjunto, pero dificulta, en gran medida, el ritmo equilibrado de la marcha del curso. La diversidad de desarrollos en el aprendizaje obliga al docente a establecer rutinas en las prácticas que provocan alteraciones en la motivación de los estudiantes. Y, sería en estos momentos en el que la implementación de actividades como las que proponemos significarían un revulsivo para reconducir la situación reactivando sus emociones.

Hemos identificado, atendiendo a diferentes motivos, tres momentos clave donde podríamos comenzar a implementar nuestra experiencia, y será en esas unidades en las que trabajaremos para diseñar los casos de estudio. El primero de ellos, en la recta final de la segunda unidad didáctica al inicio del año (entre las vacaciones de navidad y el inicio del segundo cuatrimestre). El segundo, lo planteamos con la intención de ofrecer una alternativa a las metodologías desde las que se aborda en el programa de la asignatura el ilusionismo en la representación pictórica. Y por último, el tercero, al inicio del tema dedicado a la abstracción con objeto de salvar los prejucios que suelen provocar estas prácticas artísticas en los alumnos.

En esta ocasión se seleccionaron a los artistas Santi Tena, Enrique Zabala y Oliver Johnson, por ser referentes en cada una de las materias seleccionadas, por residir en la ciudad de Valencia (un hecho que facilitaba el encuentro), y su predisposición a colaborar en nuestra iniciativa.

Por cuestiones metodológicas, nos centraremos en la presentación del proceso que hemos seguido para diseñar el primer caso de estudio, así como el desarrollo del mismo. Hemos de señalar que, teniendo en cuenta las especificidades de cada caso, el proceso se ha reeditado con Zabala y Johnson. Con Enrique Zabala pudimos organizar dos sesiones, una dedicada a conocer sus claves conceptuales y estéticas en una charla con los alumnos con la exposición de obras en clase, seguida de una masterclass donde los alumnos realizaron una aproximación a sus técnicas fotorrealista. El tercer estudio de caso, lo enfocamos desde la relación entre maestro y discípulo dentro del movimiento artístico denominado Abstracción Geométrica española motivados por la celebración en la sala Renau de la facultad de Bellas Artes de Valencia de la exposición antológica del artista internacional, pionero y antiguo profesor de la facultad, José María Yturralde. Coincidiendo con el inicio de la unidad temática dedicada a la abstracción, el pintor y antiguo colaborador de Yturralde, Oliver Johson, nos ofrecio una visión de la obra de su maestro en la sala para, seguidamente, hacer una revisión de su producción artística en un aula anexa.

\section{Caso 1: El color de los otros.}

Artista: Santi Tena.

El caso está asociado a la unidad didáctica 2, El Color. A través de la práctica el alumno se inicia en la modulación del color mediante el manejo de diferentes paletas, confección de escalas y círculos cromáticos, con el objetivo de aprehender el concepto de color en sentido 
Aprendizaje experiencial mediante estudio de casos, basados en profesionales contemporáneos, en los primeros cursos de Grado.

abstracto. Uno de los objetivos fundamentales es comprender el funcionamiento de la mezcla cromática sustractiva a través de su práctica directa. Así como, adquirir habilidades y destrezas en la modulación, armonización y contraste del color mediante la materia pictórica. Entre otras prácticas, destacan las orientadas a ensayar las mezclas por familias de pigmentos o sectores cromáticos mediante el empleo de paletas de primarios, gamas quebradas, escalas de grises cromáticos (Gage, 1993), (Garau, 1986), (Küppers, 1980), (Da Vinci, 1993).

La elección de Santi Tena obedecería fundamentalmente al empleo que el artista hace de las variables concurrentes en la especificidad del color, el tono, la saturación y la luminosidad asi como la articulación del color a partir de las variables de interdependencia cromática, el contraste y la armonía.

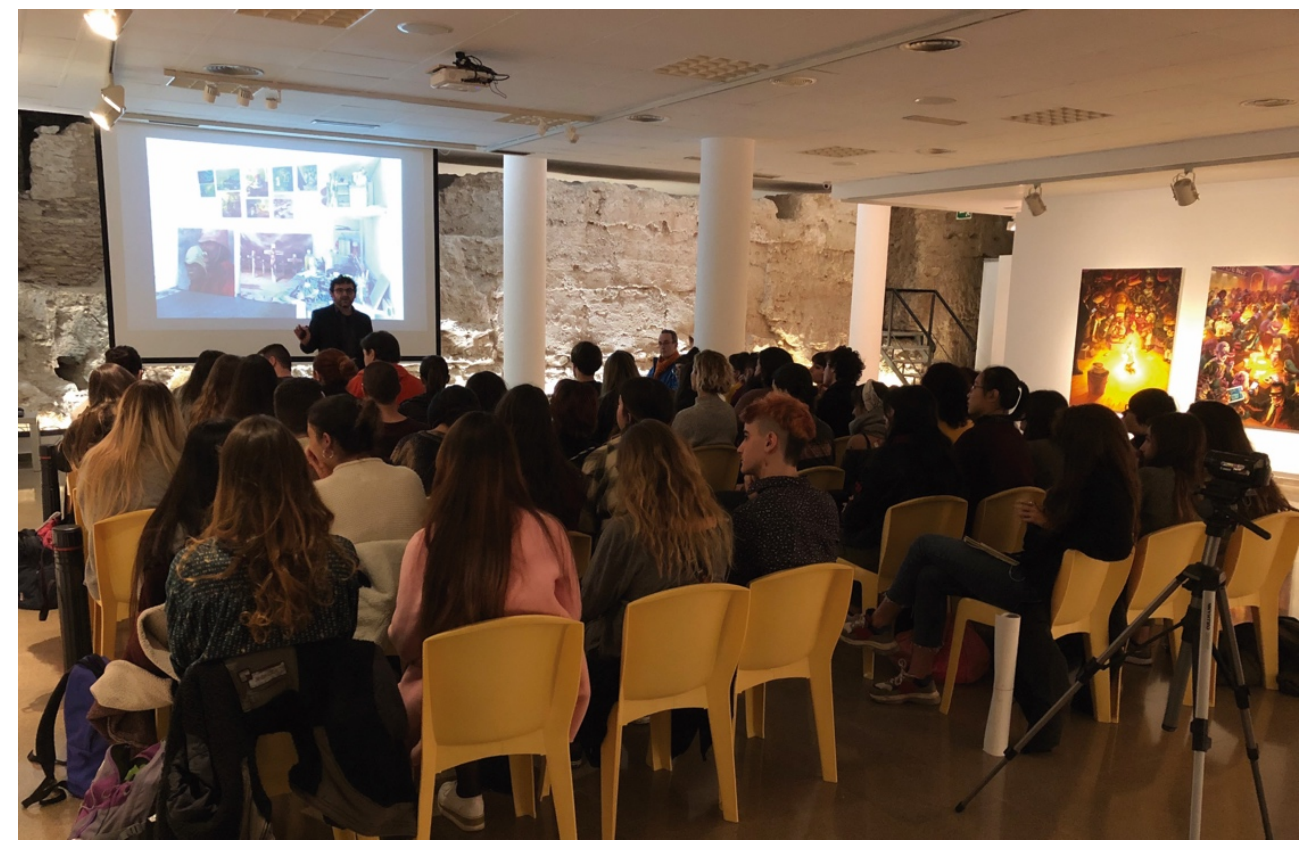

Fig. 1 Clase magistral "El color de los otros" pronunciada por Santi Tena. Sala de la Murralla. Valencia, 28/11/2018.

En esta ocasión, como en los otros dos casos, hemos aprovechado la oportunidad que nos ofreceron las circunstancias poniendo de manifiesto nuestra actitud abierta y dinámica respecto a los contenidos curriculares de la asignatura. La asistencia a la inauguración de la exposición Gastronautas de Tena en la Sala de la Muralla de Valencia nos brindo la ocasión de comprobar la relación de su obra reciente con la matería que impartía en aquel momento, aprovechando el momento para invitarlo a participar en nuestra experiencia.

En un principio le propusimos al artista la posibilidad de realizar una visita a la exposición con los alumnos donde él expusiera las claves conceptuales y estéticas de su obra asociadas a la materia. Por otro lado, le expusimos la propuesta a los alumnos para valorar su interés. La respuesta por parte de ambos no pudo ser más entusiasta, lo que nos reafirmaría en nuestros propósitos. 
A partir de ese momento comenzamos a planificar la experiencia, ya que queríamos que se convirtiera en un caso de estudio y no en otra práctica de campo. El objetivo que nos proponíamos era evidenciar las relaciones entre las prácticas realizadas por los alumnos en clase con las obras que Tena exponía en aquella muestra. De este modo, los alumnos comprobarían el sentido de sus trabajos más allá de la superación de la materia con las calificaciones obtenidas.

Una vez establecidos los contenidos se procedió al diseño de la metodología. En base a la doble condición de artista plástico-pintor y la de doctor-profesor universitario en el Grado en Maestro en Educación Infantil, se opta por el formato de clase magistral en la sala de exposiciones. Gracias a las condiciones que ofrece la sala, y la colaboración de la dirección del colegio mayor Rector Peset de la Universitat de València, se logró, por un lado, enfrentar a los alumnos a las obras en directo en el espacio expositivo, y por otro, impartir una clase magistral en el mismo espacio con el apoyo de pantalla y proyector.

Decidimos dividir la sesión de 150 minutos, el equivalente a una clase, en cuatro partes que serían conducidas y supervisadas por el profesor.

Visita a la exposición.

Contextualización del artista, por parte del profesor, dentro de la tradición pictórica occidental y como miembro activo del fenómeno Figuración Postconceptual española.

Intervención del artista con la clase magistral titulada El color de los otros, centrada en una reflexión sobre el color en su obra pictórica.

Debate con los alumnos.
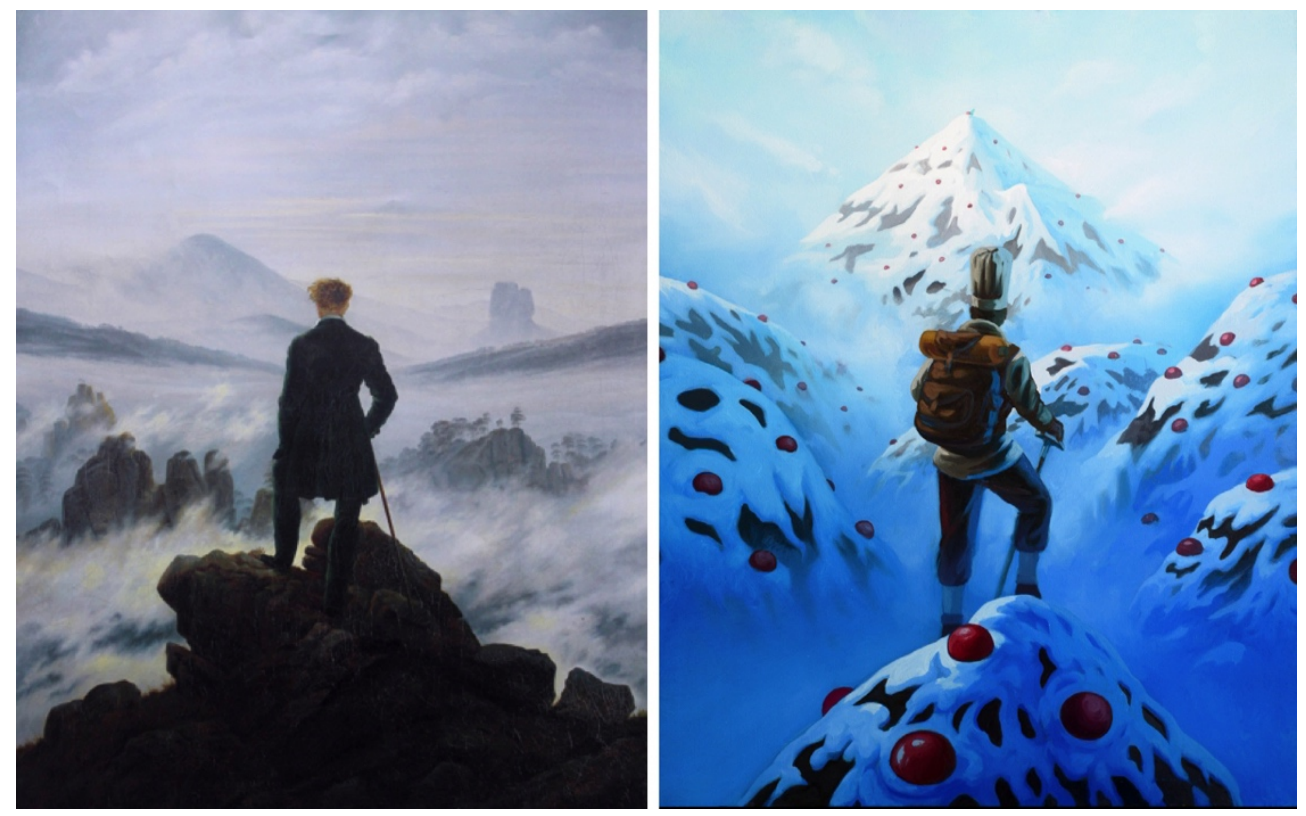

Fig. 2 Estudio de referentes: El caminante sobre el mar de nubes (1817-1818) de Caspar David Friedrich y El postre en sus manos (2018) de Santi Tena. 
Los alumnos fueron convocados en la sala de exposiciones donde los esperaba el profesor junto al artista. Los primeros treinta minutos estuvieron dedicados a visitar la exposición y a realizar observaciones sobre algunos aspectos generales de la obra.

A continuación los estudiantes tomaron asiento en las sillas situadas en el centro de la sala frente a la pantalla extensible donde se proyectaron las imágenes del documento Power Point realizado para la ocasión.

En primer lugar, el profesor realizó la contextualización partiendo de la definición de la imagen pictórica occidental en el arte renacentista para a continuación realizar un breve recorrido histórico de su desarrollo hasta las vanguardias del siglo XX, del surrealismo al pop art. Para concluir, se abordó la Figuración Postconceptual española y, más concretamente, la pintura Neometafísica valenciana, movimiento en el que se inscribe al autor, junto a Charris, Mestre, Cuéllar, De la Torre, Dis Berlin o Tomás, entre otros (De la Torre, 2012).

El color de los otros fue el título de la intervención de Santi Tena, una aproximación a su paleta cromática a partir del análisis de las claves cromáticas de la obra de sus referentes en el campo del cine, comic y pintura. Desde las películas Blade runner al la filmografía de Tim Burton, la línea clara del Herge de Tintin o artistas del arte fantástico clásicos como El Bosco o vanguardistas como Dalí. En su exposición el artista realizó un recorrido por su trayectoria, prestando una especial atención a los años de formación y a las dudas que le asaltaron en los primeros años de la carrera y como encontró respuestas en la pintura. Estableció relaciones entre sus estudios monocromáticos académicos y el papel que estas gamas juegan a la hora de despertar emociones en el espectador.

A continuación se abrió un turno de palabra en la que la gran mayoría de los estudiantes intervinieron, realizando preguntas en referencia a la técnica y temáticas de los cuadros, o a temas más generales como el mercado del arte, la promoción y la profesionalización artística. Tena respondió a todas las cuestiones durante 45 minutos.

La sesión se registró en video y se ha editado como recurso digital de la asignatura. Al terminar la sesión se les pidió a los alumnos que realizaran un One Minute Paper que entregarón a través del Poliformat de la asignatura.

\section{Resultados}

Al presentar los resultados de la aplicación de nuestra propuesta, debemos partir de la obtención de evidencias recogidas, en este caso, mediante los datos aportados por los alumnos y la observación sistemáticamente del profesor que aportará datos recogidos mediante diferentes métodos, desde el cuaderno de campo a la lista de asistencia.

En primer lugar, cabría destacar el comportamiento de los alumnos y la recepción de los contenidos de la sesión, prueba recogida en la grabación realizada de la sesión, donde se testimonia el alto grado de atención y participación de los alumnos.

Como pruebas cualitativas del grado de motivación en clase observamos mejoras en la puntualidad y asistencia, mayor dedicación en la obtención de resultados en las prácticas, dilatación en la salida del aula al concluir las clases y el empleo de referencias conceptuales y estéticas aprendidos durante el estudio del caso a la hora de argumentar sus respuestas en clase. Un cambio en la actitud de los alumnos que denota mayor interés por aprender y obtener resultados satisfactorios, según su juicio personal, que por las notas de valoración. 
En referencia al grado de aprendizaje aplicado de conceptos curriculares a través de la valoración de los trabajos, apreciamos avances en el grado de acabado, capacidad de resolución, y puesta en practica de lo aprendido con el estudio del caso, en comparación con los resultados obtenidos con anterioridad como se puso de manifiesto en las calificaciones de las prácticas realizadas con posterioridad a esta experiencia.

Como dato cuantitativo presentamos las respuestas de los alumnos recogidas en el One Minute Paper en referencia a la experiencia. Una documentación que ofrece evidencias experienciales para la evaluación de la metodología empleada respecto a aspectos, para nosotros fundamentales, como la motivación (Vivel, 2014).

A continuación presentamos una selección de extractos representativa de la valoración de la experiencia contada en primera persona por los alumnos. La información se recogió mediante la técnica del One Minute Paper al concluir la clase, con el objetivo de obtener evidencias sobre el estado real de la atención, comprensión y seguimiento de la actividad por parte del alumnado. Del mismo modo nos serviría para comprobar la adecuación efectiva de la metodología empleada a los objetivos planteados.

Las tres cuestiones fueron:

Resumen de la charla.

Valoración de la exposición.

Experiencia personal.

Destacaremos dos aspectos relevantes para nuestro estudio, por un lado la gran carga emotiva en las respuestas y el correcto empleo del lenguaje técnico aplicado al analizar la experiencia.

"La experiencia me ha resultado interesante y una manera de salir de la monotonía educativa, conociendo experiencias de otros artistas".

"Sinceramente, una actividad necesaria para todo alumno de bellas artes, una toma de contacto con la realidad, fuera de bodegones sin vida y mezclas de colores que, aparentemente, no tienen ningún sentido. Porque es entonces cuando te das cuenta que eso que haces en clase, que la mayor parte de veces casi no terminas ni de entender, aparece reflejado en obras reales, de artistas reales".

"Es motivador para nosotros ver en primera persona que alguien que ha estudiado en la misma universidad que nosotros es capaz de jugar así con la paleta. Esto nos lleva a preguntarnos a nosotros mismo si algún día seremos capaces de conseguir resultados como los de Santi”.

"Como experiencia personal he de decir que fue muy buena, la idea de dar una clase en una exposición rodeados de obras de arte me pareció un acierto absoluto, sacarnos de las clases convencionales para brindarnos la oportunidad de escuchar a un pintor hablándonos de su experiencia y de su obra en una exposición rodeados de la misma fue una experiencia muy gratificante, no me pareció una clase teórica y salí de allí habiendo aprendido muchísimas cosas. Fue una gran experiencia, espero que haya mas iguales". 
Aprendizaje experiencial mediante estudio de casos, basados en profesionales contemporáneos, en los primeros cursos de Grado.

Una vez presentados la información obtenida, debemos establecer un juicio de valor y tomar decisiones.

"La evaluación del proceso me dice que me voy acercando a la meta. Falta por analizar la última evaluación, de la que depende la orden de parada. El autor tiene que declarar si ha alcanzado su meta, si no la ha alcanzado, si debe desistir o proseguir la búsqueda" (Marina, 1996, p. 201).

Analizando la respuestas de los estudiantes, podemos comprobar como la experiencia es valorada positivamente por la gran mayoría del alumnado. Desde el punto de vista del profesor, podemos afirmar que los tres estudios de casos que hemos realizado a lo largo del curso, entre los que se incluye el de Santi Tena, han supuesto un revulsivo que ha reactivado la implicación de los estudiantes a través de la motivación.

En resumen, creemos que la experiencia del estudio de casos realizada, por su alto grado de eficacia, debería tener continuidad y ser implementada como metodología activa dentro de los contenidos de la asignatura en sintonía con las directrices del Espacio Europeo de Educación Superior.

\section{Conclusiones}

A la vista de los resultados expuestos de la práctica de metodología de casos con los alumnos de primer curso del grado de Bellas Artes 2018-19, podemos concluir que se han integrado en el programa de la asignatura a artistas contemporáneos en activo logrando evidencias de que, a través de esta metodología activa, se ha motivado a los alumnos.

Debemos señalar la circunstancia de que haber contado con la presencia de los autores en vivo ha potenciado la emoción, la comunicación de ideas, el aprendizaje, la atención,... Este factor debería tenerse en cuenta a la hora de diseñar nuevos casos. Pero cabría preguntarse si al implementar esta metodología dentro del programa de la asignatura como una actividad programada, no estaríamos perdiendo el factor sorpresa con el que hemos contado este año, debido al carácter experimental de nuestra propuesta. Y, por tanto, perdiendo la efectividad de los resultados.

También debemos apuntar que circunstancias como las acaecidas, la coincidencia temporal de la celebración de una exposición con el desarrollo de los contenidos de una unidad temática objeto de nuestro interés en el momento justo en el que entendemos que es productivo motivar al alumnado, no son habituales. En esta ocasión se han producido gracias a la casualidad, la atención por parte del docente para aprovechar las circunstancias, la complicidad de los artistas y los alumnos, sin olvidar el apoyo y colaboración del departamento de Pintura de la Universitat Politècnica de Valencia.

Estas reflexiones nos conducirían a nuestros objetivos secundarios, comenzando por el planteamiento experimental con el que hemos abordado el caso ilustrativo desde tres planteamientos diferentes, adecuándonos a las posibilidades que ofrecia el artista, la materia y el contexto. En el caso de Tena, pudimos contar con el acceso a su exposición Gastronautas e impartir la clase magistral en la misma sala. Su colaboración para preparar los contenidos e implicación en el debate con los alumnos son otros factores de carácter extraordinarios. 
Al aplicar la experiencia a los casos de Zabala y Johnson, pudimos comprobamos el carácter innovador de nuestra metodología, tanto por la capacidad de amoldarse a diferentes circunstancias como por las posibilidades que presenta para obtener la máxima eficacia. Y, también, demostrar como habríamos abierto un nuevo canal por donde introducir en la universidad el discurso de los artistas, mediante la colaboración de profesionales referentes en las materias estudiadas.

Para concluir con el repaso a nuestros objetivos, hemos presentado datos cuantitativos y cualitativos que evidenciarían, no solo, el grado de motivación y aprendizaje del estudio de casos en los alumnos, sino otros resultados positivos que no preveíamos en nuestra hipótesis inicial, expuestos anteriormente. $\mathrm{Y}$ añadir que los resultados demostrarían que estas metodologías activas pueden desarrollarse con todos los estudiantes, independientemente del curso, animando a invertir tiempo y recursos económicos para implementar estas prácticas en los primeros cursos de Grado.

Por último, deberíamos incidir en la necesidad de una reflexión previa por parte del docente para adaptar esta metodología al grupo al que va dirigido, con el objetivo de obtener un resultado favorable.

\section{Referencias}

A10TV (2019). Gastronautas. Santi Tena. [Video]. <https://youtu.be/q1uDHIJC8jw> [Consulta: 15 de marzo de 2019].

ÁlVAREZ, B., GONZÁLEZ, C. y GARCÍA, N. (2007). La motivación y los métodos de evaluación como variables fundamentales para estimular el aprendizaje autónomo. Red $U$. Revista de Docencia Universitaria, $\mathrm{n}^{\circ}$ 2. Recuperado de http://www.redu.um.es/Red_U/2 DA VINCI, L. (1993). Tratado de pintura. Madrid: Akal.

DE LA TORRE, P. (2012). Figuración Postconceptual. Valencia: Fire Drill.

EFLAND, A. D. (2002). Una historia de la educación del arte, Barcelona: Paidós.

GAGE, J. (1993). Color y cultura. Madrid: Siruela.

GARAU, A. (1986). Las armonías del color. Barcelona: Paidós.

GOLEMAN, D. (2007). Inteligencia emocional. Barcelona: Kairós.

FENANDEZ, A. (2006). Metodologías activas para la formación de competencias. Educatio siglo $X X I, \mathrm{n}^{\circ} 24$, pp. 35- 56.

KÜPPERS, H. (1980) Fundamentos de la teoría de los colores. Barcelona: Gustavo Gili.

LABRADOR, M. J., ANDREU, M. A. y GONZÁLEZ ESCRIBA, J. A. (2008). Método del caso En Labrador, M. J. \& Andreu, M. A. (eds.) Metodología Activas. Valencia: Universitat Politècnica de València, pp. 26-41.

MARINA, J. A. (1996). Teoría de la inteligencia creadora. Barcelona: Anagrama. MONROY CORNEJO, S. H. (2009) El Estudio De Caso: ¿Método o Técnica de Investigación? Revista de la Asociación Mexicana de Metodología de la Ciencia y de la Investigación, vol. $1, \mathrm{n}^{\circ} 1$. México, D. F.

$<$ http://www.ammci.org.mx/revista/pdf/Seccion\%20metodologia\%20de \%20la\%20ciencia $\% 20(1 \mathrm{a} \% 20$ parte)/EstudiodeCasoMonroy.pdf $>$ [Consulta: 15 de marzo de 2019].

SARASON, S. B. (2002). La enseñanza como arte de representación. Buenos Aires: Amorrortu. 
Aprendizaje experiencial mediante estudio de casos, basados en profesionales contemporáneos, en los primeros cursos de Grado.

SEVILLANO, M. L. (1999). Formas básicas de enseñanza-aprendizaje. En SEVILLANO, M. L. (Coord.) Estrategias de enseñanza y aprendizaje. Madrid: Universidad Nacional de Educación a Distancia, pp. 19-72.

SEVILLANO, M. L. (2011) Didáctica en el núcleo de la pedagogía. Tendencias pedagógicas, no 18 , pp. 7-32.

TORRE, S. DE LA (2009). La universidad que queremos estrategias creativas en el aula universitaria. Revista Digital Universitaria, vol. 10, $\mathrm{n}^{\circ} .12$.

ZABALZA, M.A. (2002). La enseñanza universitaria. El escenario y sus protagonistas. Madrid: Narcea.

IVAM, "Caso de estudio. Cuerpo, espacio y tiempo en Bruce Nauman". $<$ https://www.ivam.es/es/exposiciones/cuerpo-espacio-y-tiempo-en-bruce-nauman/> [Consulta: 15 de marzo de 2019].

VIVEL, M., LADO, R. y FERNÁNDEZ, S. (2014). One minute papers: rendimiento y satisfacción del alumnado. @tic. revista d'innovació educativa, no 13.

$<$ https://dialnet.unirioja.es/descarga/articulo/4951250.pdf> [Consulta: 15 de marzo de 2019]. 